\title{
Cross-Coupling of Secondary Alkyltrifluoroborates with Aryl Chlorides
}

Key words

secondary alkyltrifluoroborates

cross-coupling

aryl chlorides

parallel microscale experimentation
Significance: This valuable synthetic method demonstrates the usefulness of the parallel microscale experimentation approach in the development of new methodology in synthetic chemistry. Conditions for a smooth Pd-catalyzed crosscoupling between secondary alkyltrifluoroborates and aryl or heteroaryl chlorides have been found after the screening of a number of phosphine ligands. The parallel microscale experimentation is proved to be a very promising method of investigation in synthetic chemistry that will find more and more applications not only in industry, but also in the academic research.
Comment: The experimentation was performed in $10 \mathrm{mmol}$ scale in a 96-plate reactor, and 12 ligands were screened for each substrate, so that the optimization required much less time compared to standard optimization technique. The cross-coupling of isopropyl organometallics is often complicated by the formation of a linear product. As it is shown by the given experimental data, this side reaction takes place to a various extent with different substrate-catalyst pairs and the results are often difficult to predict. That makes a fast screening procedure for cross-coupling reactions quite desirable. 